\title{
Association Between Coronary Artery Disease and MicroRNA: Literature Review and Clinical Perspective
}

Rehan Malik ${ }^{1}$, Raja S. Mushtaque ${ }^{2}$, Usman A. Siddiqui ${ }^{3}$, Adnan Younus ${ }^{3}$, Muhammad Aziz ${ }^{4}$ , Choudhry Humayun ${ }^{5}$, Kanaan Mansoor ${ }^{3}$, Muhammad A. Latif ${ }^{6}$, Salman Waheed ${ }^{7}$, Salman Assad $^{8}$, Idrees Khan ${ }^{3}$, Syed M. Bukhari ${ }^{9}$, Daniel DelCampo ${ }^{3}$, Ali Adus ${ }^{3}$, Swetha Gannarapu 3

1. Internal Medicine, Mount Sinai Medical Center, Miami Beach, Florida,, Miami, USA 2. Medical Unit Iii, Jinnah Postgraduate Medical Center, Karachi, karachi, PAK 3. Center for Healthcare Advancement \& Outcomes Research, Baptist Health Medical Group 4. Family Medicine Center, West Kendall Baptist Hospital 5. Center for Healthcare Advancement \& Outcomes Research, Baptist Health South Florida 6. Weiss Memorial Hospital, Internal Medicine, University of Illinois at Chicago 7. Cardiology-University of Kansas Hospital \& Medical Center, University of Kansas Hospital \& Medical Center 8. Internal Medicine, Marshall University School of Medicine, Huntington, USA 9. UPMC Mckeesport Internal Medicine Department, University of Pittsburgh Medical Center

$\square$ Corresponding author: Rehan Malik,dr.ramalik@gmail.com

Disclosures can be found in Additional Information at the end of the article

\section{Abstract}

\section{Background}

Until recently, circulating micro-RNAs (miRNAs) have attracted major interest as novel biomarkers for the early diagnosis of coronary artery disease (CAD). This review article summarizes the available evidence on the correlation of micro-RNAs with both the clinical and subclinical coronary artery disease and highlights the necessity for exploring miRNAs as a potential diagnostic and prognostic biomarker of early CAD in an adult population.

\section{Methods}

A systematic literature analysis and retrieval online systems Public/Publisher MEDLINE/ Excerpta Medica Database /Medical Literature Analysis and Retrieval System Online, (PUBMED/EMBASE/MEDLINE) search were conducted for relevant information. Search was limited to the articles published in English language and conducted on humans, January 2000 onwards. We excluded studies of heart surgery, coronary artery bypass grafting (CABG), angioplasty and heart transplant. Eighteen studies met the inclusion criteria.

Received 02/20/2017

Review began 03/12/2017

Review ended 04/19/2017

Published 04/23/2017

CC Copyright 2017

Malik et al. This is an open access article distributed under the terms of the Creative Commons Attribution License CC-BY 3.0., which permits unrestricted use, distribution, and reproduction in any medium, provided the original author and source are credited.

\section{Results}

Seven out of 18 studies were multivariate, i.e. adjusted for age, gender, body mass index (BMI), smoking, hypertension, diabetes, and blood lipid profiles, while the remaining twelve studies were univariate analysis. Different sources of miRNAs were used, i.e. plasma/serum, microparticles, whole blood, platelets, blood mononuclear intimal and endothelial progenitor cells were investigated. Fourteen out of 18 studies showed up-regulation of different miRNA in $\mathrm{CAD}$ patients and in vulnerable plaque disease. Four out of 18 studies showed both the upregulation and down-regulation of miRNA in the population, while only three studies showed down-regulation of miRNA. Various sources and types of miRNA were used in each study. 


\section{Conclusion}

This review gives an extensive overview of up-regulation and down-regulation of miRNA in $\mathrm{CAD}$ and non-CAD patients. The pattern of miRNA regulation with respect to $\mathrm{CAD} /$ non-CAD study subjects varies across individual studies and different parameters, which could be the possible reason for this aberrancy. We suggest further trials be conducted in future for highlighting the role of miRNA in CAD, which may improve both the diagnostic and therapeutic approaches to stratifying $\mathrm{CAD}$ burden in the general population.

Categories: Cardiology, Family/General Practice, Internal Medicine

Keywords: mirna, coronary artery disease, association

\section{Introduction}

Heart disease is the leading cause of death for both males and females with more than half of the deaths reported in 2009 in males [1]. Coronary heart disease is the most common type of heart disease with 370,000 annual deaths, i.e. each minute someone in the United States dies from a heart disease-related event [2]. Coronary heart disease alone each year costs the United States $\$ 108.9$ billion, which includes the cost of health care services, medications, and lost productivity [3]. The total coronary artery disease (CAD) prevalence is $6.4 \%$ in US adults, which is expected to increase approximately $18 \%$ by 2030 [4]. Most individuals aged over 60 years have progressively enlarged deposits of calcium mineral in the plaques in their major arteries [5]. As atherosclerosis infiltrates the arterial wall long before it causes vessel obstruction and produces symptoms, earlier identification of this process should be part of risk prediction [6]. As such, there is a lack of cost-effective and specific biomarkers for the early clinical diagnosis and prognosis of $\mathrm{CAD}$, and there is an immense clinical demand for specific and reliable noninvasive biomarkers for CAD.

With over 1900 MicroRNA (miRNAs) discovered in humans to date, many of them have already been implicated in common human disorders. However, the pattern among the miRNA-disease association remains largely unclear for most diseases. Until recently, circulating micro-RNAs (miRNAs) have attracted major interest as novel biomarkers for the early diagnosis of CAD [7]. MiRNAs are a class of small ( $\sim 22$ nucleotides long), highly specific, endogenous, singlestranded, non-coding RNAs that regulate the expression of target genes by binding to the 39 untranslated regions and degrading or inhibiting the translation of messenger ribonucleic acid (RNA) (mRNAs) [8]. Studies have shown miRNAs' involvement in the timing of cell death and cell proliferation, hematopoiesis, and other normal cellular homeostasis [9-10]. Various miRNAs are expressed in a tissue-specific manner and thus may regulate tissue-specific functions. This review article summarizes the available evidence correlating micro-RNA, clinical and subclinical CAD and further highlights the necessity for exploring the potential of micro-RNAs as useful diagnostic and prognostic biomarkers for early CAD in the adult population.

\section{Materials And Methods}

A computerized search of the Public/Publisher MEDLINE/ Excerpta Medica Database/Medical Literature Analysis and Retrieval System Online/Excerpta Medica Database (PubMed/Medline/EMBASE) database was done with the keywords and medical subject headings (MESH) terms such as "micro RNA," "coronary artery disease," "cardiovascular disease (CVD)," "Subclinical CVD," "coronary artery calcium and micro RNA," "miRNA and high sensitivity C- reactive protein (hs-CRP)," "miRNA and coronary intimal thickness," and "miRNA and pulse wave velocity." We included all the literature that was published from January 1, 2000, until January 1, 2017. The search was limited to articles published in the 


\section{Cureus}

English language. Included studies were cross-sectional, case-control or prospective in design and conducted in adult populations (Figure 1). CAD subjects diagnosed by symptoms, imaging, cardiac enzymes, electrocardiogram (EKG), diagnostic angiography or stress testing were included. We excluded studies with CAD patients who have had heart surgery, coronary artery bypass graft $(\mathrm{CABG})$, angioplasty, and heart transplant. We also examined the references of all studies from the initial search for additional references. Demographic data was extracted from each study and results were collaborated into tables.

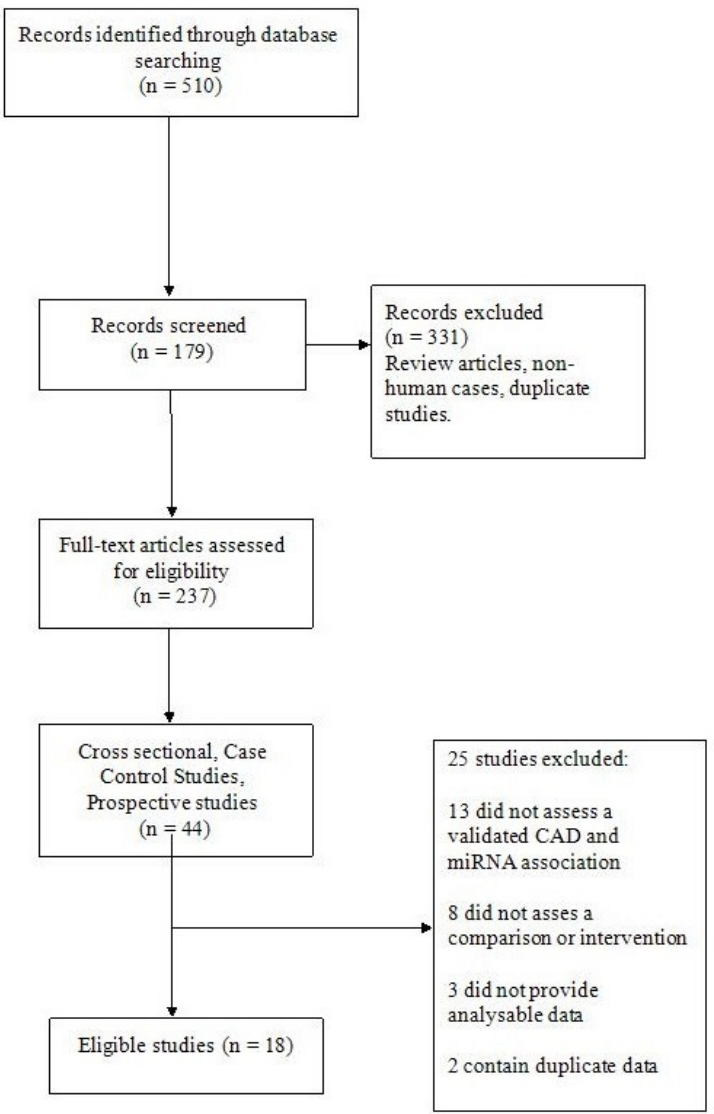

FIGURE 1: Detailed literature analysis- CAD and miRNA association

\section{Results}

A total of 18 clinical studies has been included in the review after a thorough analysis of the literature. Overall, there were 1720 subjects. The majority of the studies included were done in China, which was 11 in number, while two studies from Japan, one from the USA, one from Norway, one from Netherlands, one from the UK and one from Germany were included (Table 1). All the studies had the same outcome, which was CAD. Studies were further divided into two groups of microRNA up-regulation and down-regulation. There were four studies that checked for both up-regulation and down-regulation of microRNA. 


\section{Cureus}

\begin{tabular}{|c|c|c|c|c|c|}
\hline Serial\# & $\begin{array}{l}\text { Author, Year, Study } \\
\text { Population }\end{array}$ & Study Population Characteristics & Country & $\begin{array}{l}\text { Type } \\
\text { of } \\
\text { Study }\end{array}$ & Outcome \\
\hline 1 & $\begin{array}{l}\text { Diehl, et al.,2011 [8]; CAD } \\
(n=5), \text { ACS }(n=5)\end{array}$ & $\begin{array}{l}\text { CAD: A: } 76 \pm 2 ; \text { M: } 3(60 \%) \\
\text { ACS: A: } 66 \pm 4 ; \text { M } 5(100 \%)\end{array}$ & China & $\begin{array}{l}\text { Case } \\
\text { Control }\end{array}$ & CAD \\
\hline 2 & $\begin{array}{l}\text { Sayed, et al., } 2015 \text { [11]; } \\
\text { CAD ( } n=65) \text {, Non-CAD } \\
(n=32)\end{array}$ & $\begin{array}{l}\text { CAD: A: } 53 \text { (49-57); M: } 38 \text { (58.4\%) Non-CAD: } \\
\text { A: } 53 \text { (49-57); M: 16(50\%) }\end{array}$ & China & $\begin{array}{l}\text { Case } \\
\text { Control }\end{array}$ & CAD \\
\hline 3 & $\begin{array}{l}\text { Sayed, et al., } 2015 \text { [12]; } \\
\text { CAD }(n=37) \text {, Non-CAD } \\
(n=20)\end{array}$ & $\begin{array}{l}\text { CAD: A: } 72.97 \pm 4.28 ; \text { M: } 18(67.5 \%) \text { Non- } \\
\text { CAD: A: } 71.7 \pm 5.2, \text { M: 10(50\%) }\end{array}$ & China & $\begin{array}{l}\text { Case } \\
\text { Control }\end{array}$ & CAD \\
\hline 4 & $\begin{array}{l}\text { Zhou, et al., } 2015 \text { [13]; } \\
\text { CAD ( } n=67), \text { Non-CAD } \\
(n=67)\end{array}$ & $\begin{array}{l}\text { CAD: A } 64.70 \pm 6.79 \mathrm{M}: 43 \text { (64.2\%) Non-CAD: } \\
\text { A: } 63.69 \pm 5.96 \mathrm{M}: 32(47.8 \%)\end{array}$ & China & $\begin{array}{l}\text { Case } \\
\text { Control }\end{array}$ & CAD \\
\hline 5 & $\begin{array}{l}\text { Liu, et al., } 2015[14] ; C A D \\
(n=90) \text {, Non-CAD }(n=70)\end{array}$ & Not Given & China & $\begin{array}{l}\text { Case } \\
\text { Control }\end{array}$ & CAD \\
\hline 6 & $\begin{array}{l}\text { Han, et al., } 2015 \text { [15]; } \\
\text { CAD }(n=32) \text {, Non-CAD } \\
(n=20)\end{array}$ & $\begin{array}{l}\text { CAD A: } 67 \pm 11 ; \text { M: } 32 \text { (100\%) Non-CAD: A } \\
62 \pm 8 ; \text { M: } 20(100 \%)\end{array}$ & China & $\begin{array}{l}\text { Case } \\
\text { Control }\end{array}$ & CAD \\
\hline 7 & $\begin{array}{l}\text { Zhu, et al., } 2014[16] ; C A D \\
(n=56), \text { Non-CAD }(n=54)\end{array}$ & Not Given & China & $\begin{array}{l}\text { Case } \\
\text { Control }\end{array}$ & CAD \\
\hline 8 & $\begin{array}{l}\text { Li, et al., } 2013 \text { [17]; Pre- } \\
\text { CAD(n=12), Non-CAD } \\
(n=12)\end{array}$ & Age/Sex matched in pre CAD and Non CAD. & China & $\begin{array}{l}\text { Case } \\
\text { Control }\end{array}$ & CAD \\
\hline 9 & $\begin{array}{l}\text { Ren, et al., } 2013 \text { [18]; } \\
\text { CAD (UA) }(n=45), \text { Non- } \\
\text { CAD ( } n=37)\end{array}$ & 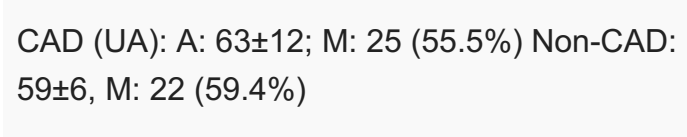 & China & $\begin{array}{l}\text { Case } \\
\text { Control }\end{array}$ & CAD \\
\hline 10 & $\begin{array}{l}\text { Guo, et al., } 2012 \text { [19]; } \\
\text { HLD+CAD ( } n=155), \text { non- } \\
\text { HLD }(n=100)\end{array}$ & $\begin{array}{l}\text { HLD+CAD: A: } 65.3 \pm 11.0 ; \text { M: } 105 \text { (67.7\%) } \\
\text { Non-CAD: A } 63.0 \pm 10.7 ; \text { M: } 35 \text { (70\%) }\end{array}$ & China & $\begin{array}{l}\text { Case } \\
\text { Control }\end{array}$ & CAD \\
\hline 11 & $\begin{array}{l}\text { Weber, et al., } 2011 \text { [20]; } \\
\text { CAD ( } n=10) \text {, Non-CAD } \\
(n=15)\end{array}$ & Age and sex matched & USA & $\begin{array}{l}\text { Case } \\
\text { Control }\end{array}$ & CAD \\
\hline 12 & $\begin{array}{l}\text { Sondermeijer, et al., } 2011 \\
{[21] \text {; Pre CAD }(n=40)} \\
\text { Non-CAD }(n=40) \text { ASCL } \\
(n=27) \text {, family }(n=40)\end{array}$ & $\begin{array}{l}\text { Age matched and males only; controls > } 20 \mathrm{yr} \\
\text { younger, sex unknown }\end{array}$ & Netherlands & $\begin{array}{l}\text { Case } \\
\text { Control }\end{array}$ & CAD \\
\hline 13 & $\begin{array}{l}\text { Fichtlscherer, et al., } 2011 \\
\text { [22];CAD ( } n=67), \text { Non- } \\
\text { CAD }(n=31)\end{array}$ & $\begin{array}{l}\text { Cohort; CAD: A: } 67.69 \pm 11.07 \text {; M: } 25(69.4 \%) \\
\text { Non-CAD: A: } 32.18 \pm 8.78 \text { Male: } 6(35.3 \%) ; \\
\text { VC; CAD: A: } 68.06 \pm 9.66 \text {; M: } 21(68 \%) \text { Non- } \\
\text { CAD: A: } 39.28 \pm 17.52 ; \text { M: 5: (36\%) }\end{array}$ & Germany & $\begin{array}{l}\text { Case } \\
\text { Control }\end{array}$ & CAD \\
\hline
\end{tabular}




\section{Cureus}

14

Taurino, et al., 2010 [23];

CAD $(n=12)$, Non-CAD

$(n=12)$

Hoesktra, et al., 2010

15

[24]; UAP $(n=25)$, SAP

$(n=25)$, Non- CAD $(n=20)$

Takahashi, et al., 2010

16

17

Li, et al., 2010 [26]; AO

$(n=104)$, Non-AO $(n=105)$

Minami, et al., 2009 [27];

18

CAD $(n=22)$

CAD: A: 66 \pm 11 ; M: 12 (100\%) Non-CAD: A:

597; M: 12 (100\%)

Age, sex, ethnically, smoking matched

CAD: A: 66.2 \pm 9.5, M: 52 (79\%) Non-CAD: A: $64.2 \pm 10.3, \mathrm{M}: 26$ (79\%)

Age Matched

CAD: A 66.1 12.8 ; M: 36 (82\%). Non-CAD: A: $64.5 \pm 8.5, \mathrm{M}: 17(77 \%)$
UK

Case

Control

CAD

Norway $\quad \begin{array}{ll}\text { Case } \\ \text { Control }\end{array}$

Japan Case

Control

CAD

Case

Control

CAD

Case

Control

\section{TABLE 1: Demographics table}

A :Age, M: Male, CAD: Coronary Artery Disease, AP: Atherosclerotic Plaque, Pre: Premature, UA: Unstable Angina, HLD: Hyperlipidemia, ACS: Acute Coronary Syndrome, ASCL: Atherosclerosis, VC: Validation Cohort, UAP: Unstable Angina Pectoris, SAP: Stable Angina Pectoris, AO : Arteriosclerosis Obliterans

\section{Up-regulation and microRNA}

A total of 15 studies reported up-regulation of micro RNAs in patients with CAD. Ten studies out of the 15 used plasma as the source while two studies used peripheral blood mononuclear cells as the source, one study used microparticles (MP) from plasma, one study used endothelial progenitor cells (EPC) and one study used platelets. The majority of the studies employed quantitative reverse transcription polymerase chain reaction (QRT-PCR) for mircoRNA analysis while only one study employed Kyoto encyclopedia of genes and genomes (KEGG) method for microRNA analysis (Li, et al. 2013). All studies reported that there is upregulation of specific miRNA in relationship to CAD. Univariate analysis was done in nine of the 15 studies, while remaining studies adjusted for age, sex, high-density lipoprotein (HDL), lowdensity lipoprotein (LDL), aspartate aminotransferase (AST), alanine aminotransferase (ALT), hypertension HTN etc in their analysis (Table 2).

\begin{tabular}{|c|c|c|c|c|c|c|c|}
\hline $\begin{array}{l}\text { Serial } \\
\#\end{array}$ & $\begin{array}{l}\text { Name of } \\
\text { Author; Year } \\
\text { of Study }\end{array}$ & $\begin{array}{l}\text { MicroRNA } \\
\text { (miR or } \\
\text { miRNA) }\end{array}$ & $\begin{array}{l}\text { MicroRNA: } \\
\text { Regulation }\end{array}$ & $\begin{array}{l}\text { MicroRNA } \\
\text { Analysis }\end{array}$ & Source & $\begin{array}{l}\text { Strength of } \\
\text { Association (Odds } \\
\text { ratio, Relative Risk or } \\
\text { Regression Analysis) }\end{array}$ & Comments \\
\hline 1 & $\begin{array}{l}\text { Diehl, et al. } \\
2012 \text { [8] }\end{array}$ & $\begin{array}{l}\operatorname{miR} 19 \mathrm{miR} \\
21 \mathrm{miR} 146 \\
\operatorname{miR} 155 \\
\operatorname{miR} 223\end{array}$ & $\begin{array}{l}\text { Up- } \\
\text { regulated: } \\
\text { miR } 19 \\
\text { miR } 21 \\
\text { miR } 146\end{array}$ & QRI-PCR & $\begin{array}{l}\text { MP } \\
\text { from } \\
\text { plasma }\end{array}$ & $\begin{array}{l}\text { ACS vs CAD miR } 21 \\
P=0.042 \mathrm{miR} 146 a \\
P=0.003\end{array}$ & Univariate Analysis \\
\hline
\end{tabular}




\section{Cureus}

\begin{tabular}{|c|c|c|c|c|c|c|c|}
\hline & & & $\begin{array}{l}\operatorname{miR} 155 \\
\operatorname{miR} 223\end{array}$ & & & & \\
\hline 2 & $\begin{array}{c}\text { Sayed, et } \\
\text { al., } 2015 \text { [11] }\end{array}$ & $\begin{array}{l}\operatorname{miR} 149 \\
\operatorname{miR} 424 \\
\operatorname{miR} 765\end{array}$ & $\begin{array}{l}\text { Up- } \\
\text { regulated: } \\
\text { miR } 765\end{array}$ & QRT-PCR & $\begin{array}{l}\text { Serum/ } \\
\text { Plasma }\end{array}$ & $\begin{array}{l}\text { Non-CAD vs. CAD } \\
\text { (Adjusted) miR } 14995 \% \\
\text { Cl } 0.894 \text { to } 0.983 p= \\
<0.0001 \text { miR } 42495 \% \\
\text { Cl } 0.863 \text { to } 0.975 p= \\
<0.0001 \text { miR } 76595 \% \\
\text { Cl } 0.939 \text { to } 0.996 \\
p=0.0001\end{array}$ & $\begin{array}{l}\text { Adjusted for age, gender, } \\
\text { TC, TAG, HDL-C, LDL-C, } \\
\text { systolic blood pressure, } \\
\text { diastolic blood pressure, } \\
\text { AST, ALT, creatinine, } \\
\text { LVEF, DM, smoking, HTN } \\
\text { and medications }\end{array}$ \\
\hline 3 & $\begin{array}{l}\text { Sayed, et al., } \\
2015 \text { [12] }\end{array}$ & $\begin{array}{l}\operatorname{miR} 149 \\
\operatorname{miR} 765\end{array}$ & $\begin{array}{l}\text { Up- } \\
\text { regulated: } \\
\text { miR } 765\end{array}$ & QRT-PCR & $\begin{array}{l}\text { Serum/ } \\
\text { Plasma }\end{array}$ & $\begin{array}{l}\text { Stable/unstable CAD vs } \\
\text { Non-CAD (Adjusted) } \\
\text { miR } 765 p=<0.001\end{array}$ & $\begin{array}{l}\text { Adjusted for subjects with } \\
\text { similar age, gender, total } \\
\text { cholesterol, total } \\
\text { glyceride, HDL, LDL, } \\
\text { systolic blood pressure, } \\
\text { diastolic blood pressure, } \\
\text { AST, ALT, creatinine, } \\
\text { cardiac troponinl, CK-MB, } \\
\text { LDH, LVEF, DM, smoking, } \\
\text { HTN, and medications }\end{array}$ \\
\hline 4 & $\begin{array}{l}\text { Zhou, et al., } \\
2015 \text { [13] }\end{array}$ & $\begin{array}{l}\operatorname{miR} 206 \\
\operatorname{miR} 574 / 5 p\end{array}$ & $\begin{array}{l}\text { Up- } \\
\text { regulated: } \\
\text { miR } 206 \\
\text { miR 574/5p }\end{array}$ & QRT-PCR & Plasma & $\begin{array}{l}\text { CAD vs Non-CAD } \\
\text { (Adjusted) miR } 206- \\
95 \% \mathrm{Cl} \text { : }(0.508-0.706) \\
\text { miR 574/5p - 95\% Cl: } \\
(0.609-0.787)\end{array}$ & $\begin{array}{l}\text { Multivariate analysis (no } \\
\text { significant differences } \\
\text { between two groups } \\
\text { including HTN, DM, } \\
\text { smoking history, age, } \\
\text { gender, HDL-C, TAG, } \\
\text { LDL-C and TC) }\end{array}$ \\
\hline 5 & $\begin{array}{l}\text { Liu, et al., } \\
2015 \text { [14] }\end{array}$ & $\begin{array}{l}\operatorname{miR} 2861 \\
\operatorname{miR} 3135 b \\
\operatorname{miR} 191 / 3 p \\
\operatorname{miR} 133 a / 3 p \\
\operatorname{miR} 1229 / 5 p \\
\operatorname{miR} 134 \\
\operatorname{miR} 3679 / 5 p \\
\operatorname{miR} 223\end{array}$ & $\begin{array}{l}\text { Up- } \\
\text { regulated: } \\
\text { miR } \\
133 a / 3 p \\
\operatorname{miR} 134 \\
\operatorname{miR} 191 / 3 p \\
\operatorname{miR} 223 \\
\operatorname{miR} \\
1229 / 5 p \\
\operatorname{miR} 2861 \\
\operatorname{miR} 3135 b \\
\operatorname{miR} \\
3679 / 5 p\end{array}$ & QRT-PCR & $\begin{array}{l}\text { Serum/ } \\
\text { Plasma }\end{array}$ & $\begin{array}{l}\text { Unadjusted miR 133a/3p } \\
95 \% \mathrm{Cl} 0.55-0.82 \mathrm{p}= \\
0.0096 \mathrm{miR} 13495 \% \mathrm{Cl} \\
0.54-0.82 \mathrm{p}=0.015 \mathrm{miR} \\
\text { 191/3p 95\% Cl 0.58- } \\
0.83 \mathrm{p}=0.0046 \mathrm{miR} 223 \\
95 \% \mathrm{Cl} 0.47-0.74 \mathrm{p}=0.13 \\
\mathrm{miR} 1229-5 \mathrm{p} 95 \% \mathrm{Cl} \\
0.54-0.83 \mathrm{p}=0.015 \mathrm{miR} \\
286195 \% \mathrm{Cl} 0.61-0.87 \\
\mathrm{p}=<0.001 \mathrm{miR} \\
3135 \mathrm{~b} 95 \% \mathrm{Cl} 0.61-0.86 \\
\mathrm{p}=<0.001 \mathrm{miR} \\
3679 / 5 \mathrm{p} 95 \% \mathrm{Cl} 0.49- \\
0.79 \mathrm{p}=0.06\end{array}$ & $\begin{array}{l}\text { Univariate Analysis } \\
\text { Matched for sex, DM, and } \\
\text { age. A key feature in } \\
\text { vasculature calcification is } \\
\text { the osteogenic transition } \\
\text { of SMCs miR } 2861 \text { might } \\
\text { function as an enhancer of } \\
\text { osteogenic differentiation } \\
\text { of SMCs. The increased } \\
\text { circulating miR } 2861 \text { level } \\
\text { may reflect CAC } \\
\text { progression. }\end{array}$ \\
\hline 6 & $\begin{array}{l}\text { Han, et al., } \\
2015 \text { [15] }\end{array}$ & $\begin{array}{l}\operatorname{miR} 21 a \\
\operatorname{miR} 23 a \\
\operatorname{miR} 34 a\end{array}$ & $\begin{array}{l}\text { Up- } \\
\text { regulated: } \\
\text { miR } 21 \\
\text { miR 23a } \\
\operatorname{miR} 34 a\end{array}$ & QRT-PCR & $\begin{array}{l}\text { Serum/ } \\
\text { Plasma }\end{array}$ & $\begin{array}{l}\text { CAD vs Non-CAD miR- } \\
34 a, \text { miR-21, and miR- } \\
23 a \text { that are differentially } \\
\text { expressed in CAD } \\
\text { plasma } p=<0.01\end{array}$ & Univariate Analysis \\
\hline & & & Up- & & & Pre CAD vs Non-CAD & \\
\hline
\end{tabular}




\section{Cureus}

\begin{tabular}{|c|c|c|c|}
\hline $\begin{array}{l}\text { Li, et al., } \\
2013 \text { [16] }\end{array}$ & miR 526b & $\begin{array}{l}\text { regulated: } \\
\text { miR 526b }\end{array}$ & $\begin{array}{l}\text { MiRNA by } \\
\text { KEGG }\end{array}$ \\
\hline $\begin{array}{l}\text { Ren, et al., } \\
2013 \text { [18] }\end{array}$ & $\begin{array}{l} \\
\\
\text { miR } 21 \text { miR } \\
25 \text { miR 92a } \\
\text { miR } 106 b \\
\text { miR } 126 \\
\text { miR } 451 \\
\text { miR 590/5p }\end{array}$ & $\begin{array}{l} \\
\text { Up- } \\
\text { regulated: } \\
\text { miR } 21 \\
\text { miR } 25 \\
\text { miR 92a } \\
\text { miR } 106 b \\
\operatorname{miR} 126 \\
\operatorname{miR} 451 \\
\operatorname{miR} 590 / 5 p\end{array}$ & QRT-PCR \\
\hline
\end{tabular}

Guo, et al., miR 122 2012 [19] miR 370

regulated: miR 122

QRT-PCR miR 370

Serum/

Plasma

Plasma

(Adjusted) miR 526b

Univariate Analysis

$p=0.00072, p=0.003215$

CAD (UA) vs Non-

CAD (Adjusted) miR

$210 \mathrm{R} 2.48895 \% \mathrm{Cl}$

$(1.173,5.277) p=<0.017$

miR 25 OR $2.036 \quad 95 \%$

Cl $(1.048,3.955) p=$

$<0.036 \mathrm{miR} 92 \mathrm{a}$ OR

$2.61195 \% \mathrm{Cl}$ (1.110,

6.144) $\mathrm{p}=<0.028 \mathrm{miR}$

106b OR $2.38995 \% \mathrm{Cl}$

Adjusted for age, sex,

$(1.158,4.927) p=<0.018$

HTN, dyslipidemia, DM,

miR 126 OR 1.882 95\%

smoking status, and the

$\mathrm{Cl}(1.140,3.108) p=$

use of statins and anti-

$<0.013 \mathrm{miR} 126 \mathrm{OR}$

1.882 95\% Cl (1.140,

3.108) $\mathrm{p}=<0.013 \mathrm{miR}$

451 OR 4.609 95\% Cl

$(2.171,9.782) p=<0.001$

miR 5905p OR

$2.67895 \% \mathrm{Cl}(1.226$,

5.849) $p=<0.013$

HLD+CAD vs Non-CAD miR 122 OR $1.0895 \%$

Serum/

Cl 1.01-1.16 $p=0.034$ miR 370 OR $1.0595 \%$ Cl $1.01-1.12 p=0.022$ platelet drugs)

Plasma

Up-

Sondermeijer,

$\operatorname{miR} 340$

[21]

$\operatorname{miR} 624$

regulated:

$\mathrm{miR} 340$

$\operatorname{miR} 624$

Pre CAD vs Non-CAD

QRT-PCR Platelets miR-340/ miR624 95\%

Cl: $0.59-0.83, p=<0.002$

miR $17 \mathrm{miR}$

92a miR Up-

Fichtlscherer, $126 \mathrm{miR}$ regulated:

11

et al., 2010

133a miR

$\operatorname{miR} 133 a$

[22]

$145 \mathrm{miR}$

miR 208a

QRT-PCR

Plasma/

CAD vs Non-CAD miR133a95\% Cl (5.22-

Serum

6.35,3.94-6.10) $p=0.16$

miR208a 95\% Cl(5.72-

$6.74,4.91-6.61) p=0.29$

$208 a$

Up-

Hokestra, et miR 135

regulated:

QRT-PCR

PBMCs

CAD vs Non-CAD miR
$135 a \mathrm{p}=<0.001$
miR 135

Univariate Analysis

Adjustment for age, gender, BMI, smoking, HTN, DM, and blood lipid profiles

Univariate Analysis

Univariate Analysis In patients with stable $C A D$, vascular-derivedMiRNAs were significantly downregulated, whereas musclederivedMiRNAs tended to be higher.

Adjusted for age, sex, culprit lesion, fasting glucose, HbA1C, LDL cholesterol, high-sensitive CRP, and history of HTN, 


\section{Cureus}

Takahashi, et al., 2010 [25]
Upregulated: $\mathrm{miR} 146 \mathrm{a} / \mathrm{b}$

\section{QRT-PCR PMBC}

CAD vs Non-CAD miR

146a/b $\quad p=<0.01$
DM, and corrected CAD.

Marked TLR4 expression

in atherosclerotic plaques, oxidative stress

upregulates macrophage

TLR4 expression, perhaps an association between

TLR4, inflammation and coronary atherosclerosis. Activation of TLR4 signal may induce miR-146a/b expression as a negative regulator and induce progression of coronary atherosclerosis.

Up-

$\mathrm{miR} 21 \mathrm{miR}$ regulated:

Li, et al., $\quad 27 \mathrm{~b} \mathrm{miR} \quad \mathrm{miR} 21$

2010 [26] $\quad$ 130, $\mathrm{miR} \quad \mathrm{miR} 27 \mathrm{~b}$

$210 \operatorname{miR} 130$

miR 210

Up-

Minami, et al., $\operatorname{miR} 221$

2009 [27] $\quad$ miR 222
Vessel

Intima $\quad A O$ vs Non-AO miR

and

Univariate Analysis

Univariate Analysis
$21 / 27 b / 130 / 210 p=<0.05$ regulated:

miR 221

miR 222
Levels of miR 221 and

miR 222 were higher in

$C A D$ group than in non-

CAD group $(p<0.01)$

\section{TABLE 2: MicroRNA up-regulated studies}

QRT-PCR: Quantitative Reverse Transcription Polymerase Chain Reaction, CAD: Coronary Artery Disease, DM: Diabetes Mellitus, HTN: Hypertension, TC: Total Cholesterol, TAG Triacylglycerols, HDL-c: High Density Lipoprotein Cholesterol, LDL-C: Low Density Lipoprotein Cholesterol, AST: Aspartate Aminotransferase, ALT: Alanine Transaminase, LVEF: Left Ventricle Ejection Fraction, CK-MB: Creatine Kinase Myocardial B fraction, LDH: Lactate Dehydrogenase, BMI: Body Mass Index, HLD : Hyperlipidemia, ACS: Acute Coronary Syndrome, KEGG: Kyoto Engenomes, SMCS: Smooth Muscle Cells, HBA1C: Hemoglobin A1C, CRP: C-Reactive Protein, TLR: Toll Like Receptor, AO: Arteriosclerosis Obliterans. EP: Endothetial Progenitor Cells, MP- Microparticles

Various different microRNA subtypes were studied with overlapping of the similar subtypes across the studies. Sayed, et al. did two different studies in two different age groups namely 4060 and geriatric population. MicroRNA 756 was significantly up-regulated in both the middleaged and elderly with a potential to be considered as a non-invasive biomarker in this population. Regulation of microRNA 223 was studied by Liu, et al. in 2015 and Diehl, et al. in 2012 in two case-control studies in China. Both the studies were not able to find a significant relationship between CAD and MiR 223, respectively. MiR 21 upregulation was tested in three of the studies: by Ren, et al. 2013, Diehl, et al., 2012 and Li, et al., 2010. Ren, et al. reported that the relationship of MiR 21 to CAD had the OR 2.488 CI 95\% (1.173-5.277) $\mathrm{p}=0.017$, while Diehl, et al. reported it to be $\mathrm{p}=0.042$ and $\mathrm{Li}$, et al. reported it to be $\mathrm{p}=0.05$. MiR 92a and 126 were under study by two authors, namely, Fichtlscherer, et al. (2010) and Ren, et al. (2013). Ren, et al. reported that miR 92a and 126 when compared with CAD and non-CAD subjects had the OR 2.611; CI 95\% (1.110-6.144) $\mathrm{p}=0.028$ and OR 1.882;CI 95\% (1.140-3.108) $\mathrm{p}=0.013$, respectively. Fichtlscherer, et al. reported MiR 92a and 126 up-regulation had no significant relationship with CAD when compared with non-CAD patients. MiR 146a was also studied by two authors 


\section{Cureus}

Takahashi, et al. and Diehl, et al. Both the authors were able to find a relationship of the upregulation of miR 146a. Lastly, miRNA 155 was also studied by two authors, namely, Fichtlscherer, et al. and Diehl, et al. Neither studies found conclusive results for the significance of this biomarker with CAD subjects when compared with non-CAD subjects.

\section{Down-regulation and mircoRNA}

A total of seven studies worked on the down-regulation of microRNA. All of the studies employed QRT-PCR as the method of analysis for detection of microRNA. Three studies used plasma as the source. Peripheral blood mononuclear cells were used by two studies. Two studies used whole blood as the source and one study used pericardial fluid and coronary arteries intima. All the studies reported down-regulation of microRNA in patients with CAD compared with non-CAD patients. The adjusted parameters were same as the up-regulation studies used.

MicroRNA subtypes which overlapped in the reviewed studies are miR 149, miR 155, and miR 145. MicroRNA 149 was studied by Sayed, et al. in 2015, in two different studies on two different age groups, which were 40-60 and a geriatric population. MicroRNA 149 was found to be down-regulated in both middle-aged and the geriatric patients. MicroRNA 155 was studied by four different authors namely, Hao, et al. 2014, Zhu, et al. 2014, Weber, et al. 2011 and Fichtlscherer, et al. Hao, et al. [28] reported 95\% CI (6.92 $\pm 0.93,5.08 \pm 1.55) \mathrm{p}=0.017$, Zhu, et al. reported by Spearmen correlation analysis $r=-0.663, p<0.001$. Weber, et al. reported relation to be significant with the $\mathrm{p}=0.002$ while Fichtlscherer, et al. reported that there was no significant relationship of microRNA 155 to CAD when compared to non-CAD patients (Table 3).

\begin{tabular}{|c|c|c|c|c|c|c|c|}
\hline & $\begin{array}{l}\text { Name of } \\
\text { Author; Year } \\
\text { of Study }\end{array}$ & $\begin{array}{l}\text { MicroRNA } \\
\text { (miR or } \\
\text { miRNA) }\end{array}$ & $\begin{array}{l}\text { MicroRNA: } \\
\text { Regulation }\end{array}$ & $\begin{array}{l}\text { MicroRNA } \\
\text { Analysis }\end{array}$ & Source & $\begin{array}{l}\text { Strength of } \\
\text { Association (Odds } \\
\text { ratio, Relative Risk } \\
\text { or Regression } \\
\text { Analysis) }\end{array}$ & Comments \\
\hline 1. & $\begin{array}{l}\text { Sayed, et al., } \\
2015 \text { [11] }\end{array}$ & $\begin{array}{l}\text { miR149 miR } \\
424 \mathrm{miR} \\
765\end{array}$ & $\begin{array}{l}\text { Down Reg: } \\
\text { miR } 149 \text { miR } \\
424\end{array}$ & QRT-PCR & $\begin{array}{l}\text { Serum/ } \\
\text { Plasma }\end{array}$ & $\begin{array}{l}\text { Non-CAD vs. CAD } \\
\text { (Adjusted) miR149 } \\
95 \% \mathrm{Cl}(0.894- \\
0.983) \mathrm{p}=0.0001 \mathrm{miR} \\
42495 \% \mathrm{Cl}(0.863- \\
0.975) \mathrm{p}=0.0001 \mathrm{miR} \\
76595 \% \mathrm{Cl}(0.939- \\
0.996) \mathrm{p}=0.0001\end{array}$ & $\begin{array}{l}\text { Adjusted for age, } \\
\text { gender, TC, TG, HDL- } \\
\text { C, LDL-C, systolic } \\
\text { blood pressure, } \\
\text { diastolic blood } \\
\text { pressure, AST, ALT, } \\
\text { creatinine, LVEF, DM, } \\
\text { smoking, HTN and } \\
\text { medications. }\end{array}$ \\
\hline 2. & $\begin{array}{l}\text { Sayed, et al., } \\
2015 \text { [12] }\end{array}$ & $\begin{array}{l}\text { miR149 miR } \\
765\end{array}$ & $\begin{array}{l}\text { Down Reg: } \\
\text { miR } 149\end{array}$ & QRT-PCR & $\begin{array}{l}\text { Serum/ } \\
\text { Plasma }\end{array}$ & $\begin{array}{l}\text { CAD vs Non CAD } \\
\text { (Adjusted) miR } 149 \\
p=0.001\end{array}$ & $\begin{array}{l}\text { Adjusted for subjects } \\
\text { with similar age, } \\
\text { gender, total } \\
\text { cholesterol, total } \\
\text { glyceride, HDL, LDL, } \\
\text { systolic blood } \\
\text { pressure, diastolic } \\
\text { blood pressure, AST, } \\
\text { ALT, creatinine, } \\
\text { cardiac troponinl, CK- } \\
\text { MB, LDH, LVEF, DM, } \\
\text { smoking, HTN, and }\end{array}$ \\
\hline
\end{tabular}




\section{Cureus}

3. Zhu, et al., 2014 [16]

miRNA 155

$\operatorname{miR} 19 a$

$\operatorname{miR} 29 a$

$\mathrm{miR} 30 \mathrm{e} / 5 \mathrm{p}$

miR 145

$\operatorname{miR} 150$

4. Weber, et al., miR 155 2011 [20]

miR 181d

miR 222

$\operatorname{miR} 342$

$\operatorname{miR} 378$

$\operatorname{miR} 584$

miR 17 miR

92a miR

Fichtlscherer, $126 \mathrm{miR}$

5 et al., 2010

[22]

133a miR

$145 \mathrm{miR}$

$155 \mathrm{miR}$

208a
Down Reg:
miR 155

Down Reg :

miR 19a miR

29a miR

$30 \mathrm{e} / 5 \mathrm{p} \mathrm{miR}$

$145 \mathrm{miR} 150$

miR 155 miR

181d miR 222

miR 342 miR

378 miR 584

Down Reg:

miR 17 miR

92a miR 126

miR 145 miR

155

Taurino,

6 et al., 2010

[23]

miR140/3p miR 140/3p

miR182 miR $182 \mathrm{miR}$

$92 \mathrm{a} / \mathrm{b}$

\section{QRT-PCR PBMC}

\section{QRT-PCR}

Whole

blood

QRT-PCR

Plasma/ 11.30,7.07-9.75) $\mathrm{p}=$ Serum

$$
\text { 9.03) }
$$
145 95\% Cl (3.87-

$5.57,4.81-5.90) p=$ 0.16

Correlation of miR155 levels in PBMCs to Gensini scores in all patients $(n=110)$. Spearman correlation analysis showed a negative correlation between miR-155 expression and the Gensini score in all patients; $r=-0.663$, $p=0.001$.

CAD vs Non-CAD

$\operatorname{miR} 19 a p=0.012$

$\operatorname{miR} 29 a p=0.012$

$\mathrm{miR} 30 \mathrm{e} / 5 \mathrm{p} p=0.02$

$\operatorname{miR} 145 p=0.008$

$\operatorname{miR} 150 p=0.006$

$\operatorname{miR} 155 p=0.002$

$\operatorname{miR} 222 p=0.001$

$\operatorname{miR} 342 p=0.001$

$\operatorname{miR} 378 p=0.001$

$\operatorname{miR} 584 p=0.036$

CAD vs Non-CAD

miR 17 95\% Cl(9.0610.97,5.42-8.29) $\mathrm{p}=0.001 \mathrm{miR} 92 \mathrm{a}$ $95 \% \mathrm{Cl}(9.48-$ $0.01 \mathrm{miR} 12695 \% \mathrm{Cl}$

Adjusted ( miR-155 was correlated to multiple metabolic and CAD RF, including age, HTN, TC, HDL-C, LDL-C, Smoking, ACEI, statins, and CRP, but not sex, hereditary and DM or Impaired Glucose Tolerance.

Univariate Analysis As whole blood samples were studied, thus miRNA profile likely reflects intracellular and extracellular miRNAs levels, in contrast to exclusively extracellular miRNAs that would be detected in plasma.

Univariate Analysis In patients with stable coronary artery disease, vascularderived miRNAs were significantly downregulated, whereas muscle derived miRNAs tended to be higher.

\section{CAD vs Non-CAD}

miR-140-3p control

vs. $C A D p=0.002$

Whole miR-182 control vs. CAD $p=0.001 \mathrm{miR}$ -

Univariate Analysis 92a/b control vs. CAD $p=0.01$

$C A D$ vs Non-CAD $\operatorname{miR} 147 p=<0.01$
Univariate Analysis 


\section{Cureus}

\section{TABLE 3: Down-Regulated microRNA studies}

Reg: Regulated, QRT-PCR: Quantitative Reverse Transcription Polymerase Chain Reaction, CAD: Coronary Artery Disease, DM: Diabetes Mellitus, HTN: Hypertension, TC: Total Cholesterol, TAG: Triacylglycerols, HDL-c: High Density Lipoprotein Cholesterol, LDLc: Low Density Lipoprotein Cholesterol, AST: Aspartate Aminotransferase, ALT: Alanine Transaminase, LVEF: Left Ventricle Ejection Fraction, CK-MB: Creatine Kinase for Myocardial B fraction, AP: Atherosclerotic Plaque, PBMC: Peripheral Blood Mononuclear Cells, RF: Risk Factor, CRP: C-Reactive Protein, ACEI: Angiotensin Converting Enzyme Inhibitor

\section{Discussion}

MiRNAs have been known to have an association with the physiological and pathological processes involved in the development of CADs such as endothelial dysfunction, inflammation, apoptosis, angiogenesis, atherosclerosis, and neointimal hyperplasia or restenosis [7,29-31]. Our systematic review summarizes 18 articles comparing miRNAs in CAD patients. Ten studies found miRNAs that were up-regulated (21-3135b), five studies showed miRNAs that were both up-regulated (21-765) and down-regulated (17-222), and three studies showed downregulated miRNAs (19a-584). miRNAs derived from plasma/serum, microparticles, whole blood, platelets, blood mononuclear intimal and endothelial progenitor cells were investigated. Circulating miRNAs (plasma or serum) exhibit remarkable stability and are highly resistant to plasma ribonuclease (RNase) activity due to internalization in macro vesicles and the formation of protein-miRNA complexes and can be used as a reliable marker for both the early diagnosis and prognosis of CAD. Therefore, the levels of individual cardiac-enriched circulating miRNAs are related to the diagnosis and prognosis of heart diseases. Recent studies have shown the involvement of miRNAs in atherosclerosis, ranging from endothelial dysfunction to plaque rupture suggesting the use of miRNA as potential biomarkers in early diagnosis of CAD [32]. miRNA upregulation or downregulation is either related to the atherosclerotic disease process or the inflammatory compensation [21].

Sayed, et al. in elderly and Zhu, et al in middle-aged documented that miRNAs (149, 424 and 155 , respectively) were down-regulated in CAD patients, Zhu, et al. postulated that miRNA 155 was downregulated because of the feedback mechanism that controls the overactivation of immune cells and thus it is negatively correlated with coronary stenosis. Similarly, Fichtlscherer, et al. found miRNA 155 to be downregulated in patients with CAD. Fichtlscherer suggested that atherosclerotic lesions uptake circulating miRNAs, thereby decreasing their levels in circulation and causing downregulation. miRNA 155 is noteworthy as it was found to be downregulated in multiple studies which used different sources (serum plasma, whole blood, and peripheral blood mononuclear cells (PMBC). Previously, Sondermeijer, et al. have reported miRNA 624 and 340 to be down-regulated in the premature CAD patients [33].

Sayed, et al., Hoekstra, et al., and Ren, et al. showed an up-regulation of miRNA 765, 134-370 and 21-5905p, respectively, in the middle-aged population of unstable CAD patients as well. Ren, et al. stated that miRNA (21-5905p) that are involved in the pathogenesis of vulnerable plaque are mostly unregulated. In these patient populations, miRNA can be used as novel biomarkers in the diagnosis of disease. The severity of the disease can be potentially seen by aberrancy of miRNA in form of either up-regulation or down-regulation. Prior studies have shown that the levels of miRNA are linked with disease severity. The up-regulated miRNA leads to the evolution of plaque towards growth, instability, and rupture [33]. Fichtlscherer, et al. in a prospective case control trial studied miRNA derived from endothelial, cardiac, skeletal, and smooth muscle cells. Interestingly, in CAD patients endothelial and smooth muscle miRNAs were up-regulated and in cardiac and skeletal muscle cells miRNA 133a/208a/155 were downregulated in the same patient population. Prior studies have also documented the differential role of miRNA in skeletal, smooth and cardiac muscle [34]. The reason for this asymmetric 
regulation is an uptake of miRNAs in cardiac muscles by atherosclerotic lesions in apoptotic bodies and endothelial cells are exposed to inflammatory cells that can increase miRNA due to cellular stressors (Fichtlscherer, et al). miRNA can be specifically monitored in certain disease processes and used as prognostic markers as seen in CAD patients with diabetes, miRNA 145 being reduced compared to those without DM (Fichtlscherer, et al). For instance, Sondermeijer, et al. showed that the platelet-derived miRNA can potentially fine-tune the expression of specific gene products that may be involved in governing platelet reactivity. Therefore, a dysfunctional miRNA-based regulatory system could lead to the development of serious platelet-related cardiovascular diseases (Sondermeijer, et al).

Studies by Zhou, et al. and $\mathrm{Li}$, et al. in CAD patients reported an upregulation of plasma-derived miRNA (140-3p/182, 206/574-5p, 134-3135b respectively). Taurino, et al. showed downregulation of whole blood-derived miRNA 92-182 at the genetic level. Interestingly, Guo, et al. and Minami, et al. in statin therapy CAD patients documented a significant downregulation of miRNA 122/370 and 221/222 levels that were previously up-regulated. Endothelial progenitor cells play an important level and in the regulation of miRNA after treatment with lipid therapy on lipometabolism related miRNA (Minami, et al.). Similarly, Takahashi, et al. noted a decrease in the miRNA $146 \mathrm{a}-\mathrm{b}$ levels in CAD patients in post-statin and angiotensin-converting enzyme inhibitor/angiotensin receptor blocker (ACEI/ARB) therapy as well. In a cross-sectional study by Weber, et al., miRNA 19a-584 was also reported to be significantly down-regulated in CAD patients post ACE/ARB therapy. The response of miRNA to medications may provide an insight into the prognostic value of miRNA. Pharmacological therapy has been known to have an effect through gene signaling pathway, which further influences miRNA levels [7]. Diehl, et al. examined microparticles derived miRNA 19-223 and documented an upregulation in acute coronary syndrome (ACS) patients. Certain miRNA are shown to be involved in cardiac hypertrophy and angiogenesis and shown to be up-regulated in microparticles (Diehl, et al). Interestingly, Li, et al. observed upregulation of miRNA 21-210 in the intima of arteriosclerosis obliterans (ASO) patients. miRNA has been shown to be proangiogenic through its effects at the genetic level (Li, et al).

\section{Limitations}

Firstly, criteria for CAD participant enrolment across the studies were not standardized. Secondly, the study cohorts were not age and sex matched. Thirdly, the population size of different trials was very small. Fourthly, none of the included studies prospectively analyzed a specific miRNA regulation derived from one source or from different sources. Even specific miRNA regulation can be varied when compared across different studies and sources of origin. The pre and post CAD miRNA levels were not mentioned by any of the studies. Large randomized double-blinded studies are needed to eliminate population bias and increase the statistical power of each study. Prospective case-control trials with longer followup periods may play a role in deciphering the prognostic potential for the miRNA markers in CAD patients. Lastly, studies included did not mention specifically the number of patients on ACE/ARBs or statins.

\section{Conclusions}

miRNA expression profile is associated with several human cardiovascular diseases, suggesting their role as a novel class of biomarkers as well as potential treatment targets for cardiovascular diseases. This systematic review provides a potential insight on miRNA regulations in subjects with and without CAD and highlights the different parameters which could be the reason for aberrant miRNA expression. Further understanding of miRNA expression may help to delineate their role in improving both the diagnostic and therapeutic approaches to stratifying CAD burden in the general population. 


\section{Additional Information \\ Disclosures}

Human subjects: All authors have confirmed that this study did not involve human participants or tissue. Animal subjects: All authors have confirmed that this study did not involve animal subjects or tissue. Conflicts of interest: In compliance with the ICMJE uniform disclosure form, all authors declare the following: Payment/services info: All authors have declared that no financial support was received from any organization for the submitted work. Financial relationships: All authors have declared that they have no financial relationships at present or within the previous three years with any organizations that might have an interest in the submitted work. Other relationships: All authors have declared that there are no other relationships or activities that could appear to have influenced the submitted work.

\section{References}

1. Underlying cause of death. (1999-2003). Accessed: 2015: http://wonder.cdc.gov/ucdicd10.html.

2. Mozaffarian D, Benjamin EJ, Go AS, et al.: Heart disease and stroke statistics-2015 update: a report from the American Heart Association . Circulation . 2015, 131:29-322. 10.1161/CIR.0000000000000152

3. Centers for Disease Control and Prevention (CDC): Million hearts: strategies to reduce the prevalence of leading cardiovascular disease risk factors-United States, 2011. MMWR Morb Mortal Wkly Rep. 2011, 60:1248-51.

4. Go AS, Mozaffarian D, Roger VL, Benjamin EJ, et al.: Heart disease and stroke statistics-2014 update: a report from the American Heart Association. Circulation . 2014, 129:28-292. 10.1161/01.cir.0000441139.02102.80

5. Demer LL, Tintut Y: Vascular calcification: pathobiology of a multifaceted disease . Circulation. 2008, 117:2938-48. 10.1161/CIRCULATIONAHA.107.743161

6. Shaw LJ, Raggi P, Berman DS, et al.: Cost-effectiveness of screening for cardiovascular disease with measures of coronary calcium. Prog Cardiovasc Dis. 2003, 46:171-84. 10.1016/S00330620(03)00085-9

7. Gupta SK, Bang C, Thum T: Circulating microRNAs as biomarkers and potential paracrine mediators of cardiovascular disease. Circ Cardiovasc Genet. 2010, 3:484-488. 10.1161/CIRCGENETICS.110.958363

8. Diehl P, Fricke A, Sander L, et al.: Microparticles: major transport vehicles for distinct microRNAs in circulation. Cardiovasc Res. 2012, 93:633-44.

9. Slezak-Prochazka I, Durmus S, Kroesen BJ, et al.: MicroRNAs, macrocontrol: regulation of miRNA processing. RNA. 2010, 16:1087-95. 10.1261/rna.1804410

10. Bartel DP: MicroRNAs: genomics, biogenesis, mechanism, and function. Cell. 2004, 116:28197. 10.1016/S0092-8674(04)00045-5

11. Md Sayed AS, Xia K, Li F, et al.: The diagnostic value of circulating microRNAs for middleaged (40-60-year-old) coronary artery disease patients. Clinics. 2015, 70:257-263.

12. Sayed ASM, Xia K, Li F, et al.: Circulating miR-765 and miR-149: potential noninvasive diagnostic biomarkers for geriatric coronary artery disease patients. Biomed Res Int. 2015, 2015:1-8. 10.1155/2015/740301

13. Zhou J, Shao G, Chen X, et al.: MiRNA 206 and miRNA 574-5p are highly expression in coronary artery disease. Biosci Rep. 2016, 1:295. 10.1042/BSR20150206

14. Liu W, Ling S, Sun W, et al.: Circulating microRNAs correlated with the level of coronary artery calcification in symptomatic patients. Sci Rep . 2015, 5:16099. 10.1038/srep16099

15. Han H, Qu G, Han C, et al.: MiR-34a, miR-21 and miR-23a as potential biomarkers for coronary artery disease: a pilot microarray study and confirmation in a 32 patient cohort. Exp Mol Med. 2015, 47:138. 10.1038/emm.2014.81

16. Zhu GF, Yang LX, Guo RW, et al.: MicroRNA-155 is inversely associated with severity of coronary stenotic lesions calculated by the Gensini score. Coron Artery Dis. 2014, 25:304-10. 10.1097/MCA.0000000000000088

17. Li K, Zhang T, Fan H, et al. : The analysis of microRNA expression profiling for coronary 
artery disease. Cardiology. 2014, 127:62-9. 10.1159/000354872

18. Ren J, Zhang J, Xu N, et al.: Signature of circulating microRNAs as potential biomarkers in vulnerable coronary artery disease. PLoS One. 2013, 8: 10.1371/journal.pone.0080738

19. Guo W, Liu H, Li L, et al.: Regulation of lovastatin on a key inflammation-related microRNA in myocardial cells. Chin Med J (Engl). 2014, 127:2977-81.

20. Weber M, Baker MB, Patel RS, et al.: MicroRNA expression profile in CAD patients and the impact of ACEI/ARB. Cardiol Res Pract. 2011, 2011:1-5. 10.4061/2011/532915

21. Sondermeijer BM, Bakker A, Halliani A, et al.: Platelets in patients with premature coronary artery disease exhibit upregulation of miRNA340* and miRNA624*. PLoS One . 2011, 6:25946. 10.1371/journal.pone.0025946

22. Fichtlscherer S, De Rosa S, Fox H, et al.: Circulating microRNAs in patients with coronary artery disease. Circ Res . 2010, 107:677-84. 10.1161/CIRCRESAHA.109.215566

23. Taurino C, Miller WH, McBride MW, et al.: Gene expression profiling in whole blood of patients with coronary artery disease . Clin Sci . 2010, 119:335-43. 10.1042/CS20100043

24. Hoekstra M, van der Lans CA, Halvorsen B, et al.: The peripheral blood mononuclear cell microRNA signature of coronary artery disease. Biochem Biophys Res Commun. 2010, 394:792-7. 10.1016/j.bbrc.2010.03.075

25. Takahashi Y, Satoh M, Minami Y, et al.: Expression of miR-146a/b is associated with the Tolllike receptor 4 signal in coronary artery disease: effect of renin-angiotensin system blockade and statins on miRNA-146a/b and Toll-like receptor 4 levels. Clin Sci . 2010, 119:395-405. $10.1042 / C S 20100003$

26. Li T, Cao H, Zhuang J, Wan J, et al.: Identification of miR-130a, miR-27b and miR-210 as serum biomarkers for atherosclerosis obliterans. Clin Chim Acta. 2011, 412:66-70. 10.1016/j.cca.2010.09.029

27. Minami Y, Satoh M, Maesawa C, et al.: Effect of atorvastatin on microRNA 221 / 222 expression in endothelial progenitor cells obtained from patients with coronary artery disease . Eur J Clin Invest. 2009, 39:359-67. 10.1111/j.1365-2362.2009.02110.x

28. Hao L, Wang XG, Cheng JD, et al.: The up-regulation of endothelin-1 and down-regulation of miRNA-125a-5p, -155, and -199a/b-3p in human atherosclerotic coronary artery. Cardiovasc Pathol. 2014, 23:217-23. 10.1016/j.carpath.2014.03.009

29. Small EM, Olson EN: Pervasive roles of microRNAs in cardiovascular biology . Nature. 2011, 469:336-42. 10.1038/nature09783

30. Nishiguchi T, Imanishi T, Akasaka T: MicroRNAs and cardiovascular diseases. Biomed Res Int. 2015, 2015:682857. 10.1155/2015/682857

31. Dangwal S, Bang C, Thum T: Novel techniques and targets in cardiovascular microRNA research. Cardiovasc Res. 2012, 93:545-54. 10.1093/cvr/cvr297

32. Maitrias P, Metzinger-Le Meuth V, Massy ZA, et al.: MicroRNA deregulation in symptomatic carotid plaque. J Vasc Surg. 2015, 62:1245-1250. 10.1016/j.jvs.2015.06.136

33. Comer BS, Camoretti-Mercado B, Kogut PC, et al.: MicroRNA-146a and microRNA-146b expression and anti-inflammatory function in human airway smooth muscle. Am J Physiol Lung Cell Mol Physiol. 2014, 307:727-34. 10.1152/ajplung.00174.2014

34. Kim JY, Park YK, Lee KP, et al.: Genome-wide profiling of the microRNA-mRNA regulatory network in skeletal muscle with aging. Aging. 2014, 6:524-44. 10.18632/aging.100677 